\title{
Modelling the Nonlinear Wave Motion within the Scope of the Fractional Calculus
}

\author{
Abdon Atangana, ${ }^{1}$ Seyma Tuluce Demiray, ${ }^{2}$ and Hasan Bulut ${ }^{2}$ \\ ${ }^{1}$ Institute for Groundwater Studies, Faculty of Natural and Agricultural Sciences, University of the Free State, \\ Bloemfontein 9300, South Africa \\ ${ }^{2}$ Department of Mathematics, Faculty of Science, Firat University, 23119 Elazig, Turkey
}

Correspondence should be addressed to Abdon Atangana; abdonatangana@yahoo.fr

Received 26 March 2014; Revised 5 May 2014; Accepted 6 May 2014; Published 22 May 2014

Academic Editor: Ali H. Bhrawy

Copyright (C) 2014 Abdon Atangana et al. This is an open access article distributed under the Creative Commons Attribution License, which permits unrestricted use, distribution, and reproduction in any medium, provided the original work is properly cited.

The aim of this paper was to first extend the model describing the nonlinear wave movement to the concept of noninteger order derivative. The extended equation was investigated within the scope of an iterative method. The stability and convergence analysis of the iteration method for this extended equation was presented in detail. The uniqueness of the special solution was also investigated. A resume of the method for solving this equation was provided. The algorithm was used to derive the unique special solution for given initial conditions.

\section{Introduction}

The Kadomtsev-Petviashvili equation (or basically the KP equation) is a nonlinear partial differential equation in two spatial and one temporal coordinates that illustrate the development of nonlinear, extended waves of diminutive amplitude with sluggish reliance on the crosswise coordinate. There are two dissimilar descriptions of the KP equation. Nevertheless the version we are interesting in is given below as

$$
\partial_{x}\left(\partial_{t} u+u \partial_{x} u+\varepsilon^{2} \partial_{x x x} u\right)+\lambda \partial_{y y} u=0 .
$$

The case $\lambda=1$ is known as the KPII equation and models, for instance, water waves with small surface tension. The case $\lambda=i$ is known as the KPI equation and may be used to model waves in thin films with high surface tension [1-4]. The equation is frequently in black and white with dissimilar coefficients in front of the various terms, but the particular values are inessential, since they can be modified by appropriately rescaling the dependent and independent variables [5]. The $\mathrm{KP}$ equation is a worldwide integrable structure in two spatial dimensions in the similar line of attack that the KdV equation can be looked upon as a widespread integrable system in one spatial dimension, since many other integrable systems can be obtained as reductions [5]. Per se, the KP equation has been comprehensively considered in the mathematical society in the last forty years. The KP equation is also one of the generally common models in nonlinear wave theory, which arises as a reduction of system with quadratic nonlinearity, which admits weakly dispersive waves, in a paraxial wave approximation [3]. The equation unsurprisingly comes into view as a well-known boundary in the asymptotic explanation of such systems in which merely the most important order terms are engaged and an asymptotic equilibrium flanked by weak scattering, quadratic nonlinearity and diffraction is supposed. The poles separately acted by the two spatial variables accounts for the asymmetric way in that they appear in the equation [4].

In the last decade, many physical problems have been with great success described within differential equations with both integer order and fractional order. More importantly it was observed most of the time that, all physical problems described or modelled within the folder of the fractional order derivative were more predictable than the conventional order derivative, for instance, in the study of groundwater problems [6-10] and others [11-15]. One aspect of this study is to revert the conventional KP equation to the concept of fractional order derivative. However one of 
the big problems encountered in modelling real world problems is perhaps to find the solution of these nonlinear equations, especially when it came to fractional differential equation. Another aspect of this paper will therefore be devoted to the derivation of approximation of the generalized $\mathrm{KP}$ equation; we will also present the convergence and the uniqueness of the special solution. The generalised equation under study here is given as

$$
\begin{array}{r}
\partial_{t x}^{\alpha+1} u+\left(\partial_{x} u\right)^{2}+u \partial_{x x} u+\varepsilon^{2} \partial_{x x x x}^{4} u+\lambda \partial_{y y}^{2} u=0, \\
0<\alpha \leq 1 .
\end{array}
$$

We will present some basic formulas of the noninteger derivative in the following sections.

\section{Basic Knowledge about Fractional Derivatives}

One can find nowadays in the literature different definitions of fractional derivatives. However the furthermost well-liked ones are the Riemann-Liouville and the Caputo derivatives. For Caputo we have

$$
{ }_{0}^{C} D_{x}^{\alpha}(f(x))=\frac{1}{\Gamma(n-\alpha)} \int_{0}^{x}(x-t)^{n-\alpha-1} \frac{d^{n} f(t)}{d t^{n}} d t .
$$

For the case of Riemann-Liouville we have the following definition:

$$
D_{x}^{\alpha}(f(x))=\frac{1}{\Gamma(n-\alpha)} \frac{d^{n}}{d x^{n}} \int_{0}^{x}(x-t)^{n-\alpha-1} f(t) d t .
$$

Each one of these fractional derivatives presents some compensations and weakness [16-18].

Definition 1. Let $\Omega=[a, b](-\infty \leq a<b \leq \infty)$ be a finite or infinite interval of real axis $\mathbb{R}=(-\infty, \infty)$. We denote by $L_{p}(a, b)(1 \leq p \leq \infty)$ the set of those Lebesgue complexvalued measurable functions $f$ on $\Omega$ for which $\|f\|_{p}<\infty$, where

$$
\|f\|_{p}=\left(\int_{a}^{b}|f(t)|^{p} d t\right)^{1 / p} \quad(1 \leq p \leq \infty) .
$$

We will, in addition to the above definition, present the following useful theorem.

Theorem 2. If $h(t) \in L_{1}(\mathbb{R})$ and $h_{1}(t) \in L_{p}(\mathbb{R})$, then their convolution $\left(h * h_{1}\right)(x) \in L_{p}(\mathbb{R})(1 \leq p \leq \infty)$, and the following inequality holds [16]:

$$
\left\|f\left(h * h_{1}\right)(x)\right\|_{p}<\|h\|_{1}\left\|h_{1}\right\|_{p} .
$$

In particular, if $h(t) \in L_{1}(\mathbb{R})$ and $h_{1}(t) \in L_{2}(\mathbb{R})$, then their convolution $\left(h * h_{1}\right)(x) \in L_{2}(\mathbb{R})$; then,

$$
\left\|f\left(h * h_{1}\right)(x)\right\|_{2}<\|h\|_{1}\left\|h_{1}\right\|_{2} .
$$

Lemma 3 (see [16]). The fractional integration operators with $\mathfrak{R}(\alpha)>0$ are bounded in $L_{p}(a, b)(1 \leq p \leq \infty)$ :

$$
\left\|I_{a}^{\alpha} f\right\|_{p} \leq K\|f\|_{p}, \quad K=\frac{(b-a)^{\mathfrak{R}(\alpha)}}{\mathfrak{R}(\alpha)|\Gamma(\alpha)|} .
$$

\section{Derivation of the Special Solution}

The real problem with nonlinear equation is to find a suitable analytical method that can be used to derive their exact or special solutions. No wonder, many scholars have devoted their attention in developing methods to solve these equations analytically or numerically, but when the partial differential equation with mixed derivative is concerned no well-accurate approach has been proposed. We will in this paper propose an iteration method to derive the fractional Kadomtsev-Petviashvili equation.

The method proposed here consists of applying first the inverse operator of $\partial_{t x}^{\alpha+1}$ on both sides of (2) to obtain the following:

$$
\begin{aligned}
& u(x, y, t) \\
& =u(x, y, 0)+u(0, y, t)-u(0, y, 0) \\
& +\frac{1}{\Gamma(\alpha)} \int_{0}^{x} \int_{0}^{t}(t-l)^{\alpha-1}\left(\left(\partial_{v} u\right)^{2}+u \partial_{v v} u\right. \\
& \left.\quad+\varepsilon^{2} \partial_{v v v v}^{4} u+\lambda \partial_{y y}^{2} u\right) d v d l
\end{aligned}
$$

To solve the above equation, we assume that its solution is in series form as

$$
u(x, y, t)=\sum_{n=0}^{\infty} u_{n}(x, y, t)
$$

Secondly we introduce an artificial parameter according to the concept of the homotopy, now after substituting (10) into (9) and putting together terms of same power of the artificial parameter $p$, we arrive at the following equations:

$$
\begin{aligned}
& u_{0}(x, y, t)=u(x, y, 0)+u(0, y, t)-u(0, y, 0) \\
& u_{1}(x, y, t) \\
& =\frac{1}{\Gamma(\alpha)} \int_{0}^{x} \int_{0}^{t}(t-l)^{\alpha-1}\left(\left(\partial_{v} u_{0}\right)^{2}+u \partial_{v v} u_{0}\right. \\
& \left.+\varepsilon^{2} \partial_{v v v v}^{4} u_{0}+\lambda \partial_{y y}^{2} u_{0}\right) d v d l \\
& u_{n}(x, y, t) \\
& =\frac{1}{\Gamma(\alpha)} \int_{0}^{x} \int_{0}^{t}(t-l)^{\alpha-1}\left(H_{n-1}^{1}+H_{n-1}^{2}+\varepsilon^{2} \partial_{v v v v}^{4} u_{n-1}\right. \\
& \left.+\lambda \partial_{y y}^{2} u_{n-1}\right) d v d l, \quad n \geq 1, \\
& H_{n-1}^{1}=\sum_{k=0}^{n-1} \partial_{\nu} u_{k} \partial_{v} u_{n-k-1} \\
& H_{n-1}^{2}=\sum_{k=0}^{n-1} \partial_{v} u_{k} u_{n-k-1}
\end{aligned}
$$

The iteration formula in (11) can be used to derive all terms providing that the initial conditions are given. We will put 
the above procedure in a resume usually called algorithm to help readers of the numerical method field implement method via computer.

Algorithm 4. Consider

(i) input: $I(x, y, t)=u(x, y, 0)+u(0, y, t)-u(0, y, 0)$ as early boarder,

(ii) $k$-number terms in the series calculation,

(iii) output: $u_{\text {part }}(x, y, t)$, the special solution.

Step 1. Put $u_{0}(x, y, t)=I(x, y, t)$ and $u_{\text {part }}(x, y, t)=$ $u_{0}(x, y, t)$.

Step 2. For $k=0$ to $n-1$ do Steps 3, 4, and 5 .

Step 3. Compute

$$
\begin{aligned}
H_{n-1}^{1}= & \sum_{k=0}^{n-1} \partial_{v} u_{k} \partial_{v} u_{n-k-1}, \\
H_{n-1}^{2}= & \sum_{k=0}^{n-1} \partial_{v} u_{k} u_{n-k-1}, \\
& b_{n}=\frac{1}{\Gamma(\alpha)} \\
& \quad \times \int_{0}^{x} \int_{0}^{t}(t-l)^{\alpha-1}\left(H_{n-1}^{1}+H_{n-1}^{2}+\varepsilon^{2} \partial_{v v v v}^{4} u_{n-1}\right. \\
& \left.+\lambda \partial_{y y}^{2} u_{n-1}\right) d v d l, \quad n \geq 1 .
\end{aligned}
$$

Step 4. Compute

$$
u_{n+1}(x, t)=v_{n}+u_{n}
$$

Step 5. Compute

$$
u_{\text {part }}(x, y, t)=u_{\text {part }}(x, y, t)+u_{n+1}(x, y, t) .
$$

Stop.

\section{Convergence and Uniqueness Analysis of the Proposed Method}

In recent years, many papers have been published in which iterations methods are used to give approximate solution to the nonlinear equations. However in these papers, no study of stability, the convergence, and the uniqueness analysis has been done. This has become nowadays a routine whereas the study of convergence is very important and very difficult because the proof of convergence shows the strength of the method. We therefore devote this section to the study of the convergence of the scheme used here and applied to the fractional nonlinear wave motion. To achieve this we will think about the following fractional sub-Hilbert space $H_{\alpha}$ of the Hilbert space $H=L^{2}((a, b) \times(0, T))$ [19] that can be defined as the set of those functions:

$$
\begin{gathered}
v:(a, b) \times[0, T] \longrightarrow \mathbb{R}, \\
\frac{1}{\Gamma(\alpha)} \iint(t-l)^{\alpha-1} v^{2} d l d s<\infty .
\end{gathered}
$$

We correspondingly undertake that the differential operatives are circumscribed under the $L^{2}$ norms. We consider the fractional Kadomtsev-Petviashvili equation in the light of the above assumptions and then the operator form of the equation is

$$
B(u)=-\partial_{t x}^{\alpha+1}(u)=\left(\partial_{x} u\right)^{2}+u \partial_{x x} u+\varepsilon^{2} \partial_{x x x x}^{4} u+\lambda \partial_{y y}^{2} u .
$$

The homotopy decomposition method used here is convergent if the shadowing two suggestions are fulfilled (see [20] and references therein).

(H1) $(B(u)-B(v), u-v) \leq q\|u-v\|, q>0$ for all $(u, v) \in H_{\alpha}$.

(H2) For any positive constant $M>0$ there exists a constant $P>0$ such that, for $u, v \in H$ with $\|u\| \leq$ $M,\|v\| \leq M$.

We have

$$
(B(u)-B(v), w) \leq P\|u-v\|\|w\| \quad \forall w \in H_{\alpha} .
$$

We will present some properties of the inner product.

4.1. Properties of the Inner Product [21, 22]. The commentary on Hilbert space entertains numerous illustrations of the inner product spaces wherein the metric produced by the inner product profits a complete metric space. Inner product spaces have an instinctively outlined norm grounded upon the inner product of the space itself that does not the parallelogram equality:

$$
\|x\|=\sqrt{(x, x)} .
$$

It is well defined by the no negativity axiom of the definition of the inner product space. The following properties can be observed:

$$
|(x, y)| \leq\|x\|\|y\| .
$$

The above is the well-known Cauchy-Schwarz inequality. Also the following can be obtained:

$$
\|a \cdot x\|=|a| \cdot\|x\| .
$$

The above is called homogeneity. The last interesting one for this paper will be given as

$$
\|x+y\| \leq\|x\|+\|y\| .
$$

The above is called triangular inequality. 
Proof. Using the definition of our operator $B$, we have the following:

$$
\begin{aligned}
B(u)-B(v)= & \left(\partial_{x} u\right)^{2}-\left(\partial_{x} v\right)^{2}+u \partial_{x x} u-v \partial_{x x} v \\
& +\varepsilon^{2} \partial_{x x x x}^{4}(u-v)+\lambda \partial_{y y}^{2}(u-v) \\
= & \partial_{x}\left(u \partial_{x} u-v \partial_{x} v\right)+\varepsilon^{2} \partial_{x x x x}^{4}(u-v) \\
& +\lambda \partial_{y y}^{2}(u-v) \\
= & \partial_{x}\left(\frac{\partial_{x} u^{2}-\partial_{x} v^{2}}{2}\right)+\varepsilon^{2} \partial_{x x x x}^{4}(u-v) \\
& +\lambda \partial_{y y}^{2}(u-v) .
\end{aligned}
$$

With the above reduction in hand, we can now proceed to compute

$$
\begin{aligned}
& (B(u)-B(v), u-v) \\
& =\left(\partial_{x}\left(\frac{\partial_{x} u^{2}-\partial_{x} v^{2}}{2}\right), u-v\right)+\left(\varepsilon^{2} \partial_{x x x x}^{4}(u-v), u-v\right) \\
& \quad+\left(\lambda \partial_{y y}^{2}(u-v), u-v\right) .
\end{aligned}
$$

We will consider case by case the components of the above equation:

$$
\left(\partial_{x}\left(\frac{\partial_{x} u^{2}-\partial_{x} v^{2}}{2}\right), u-v\right)
$$

Regarding the bodily problem under examination, we are required to point out that the wave can only be propagated in finite space; therefore, $u, v$ are bordered, as a result, we can find a positive constant $N$ such that $(u, u),(v, v)<N^{2}$. It follows by making use of Schwartz inequality that

$$
\left(\partial_{x}\left(\frac{\partial_{x} u^{2}-\partial_{x} v^{2}}{2}\right), u-v\right) \leq\left\|\partial_{x}\left(\frac{\partial_{x} u^{2}-\partial_{x} v^{2}}{2}\right)\right\|\|u-v\| .
$$

Using the properties of the norm and the derivative, we can possibly find a positive real number, say, for instance, $O_{1}$, satisfying

$$
\left\|\partial_{x}\left(\frac{\partial_{x} u^{2}-\partial_{x} v^{2}}{2}\right)\right\| \leq O_{1}\left\|\frac{\partial_{x} u^{2}-\partial_{x} v^{2}}{2}\right\| .
$$

Using the same properties, we can get hold of a second positive real constant, say, for instance, $\mathrm{O}_{2}$, that will allow us to have

$$
\left\|\partial_{x}\left(\frac{\partial_{x} u^{2}-\partial_{x} v^{2}}{2}\right)\right\| \leq \frac{O_{1} O_{2}}{2}\left\|u^{2}-v^{2}\right\| .
$$

We can now further extend this to the following:

$$
\left\|\partial_{x}\left(\frac{\partial_{x} u^{2}-\partial_{x} v^{2}}{2}\right)\right\| \leq \frac{O_{1} O_{2}}{2}\|u-v\|\|u+v\| .
$$

Now we can use the fact that the nonlinear wave is propagating in the finite space to obtain $\|u-v\|\|u+v\| \leq 4 N^{4}$ such that

$$
\left(\partial_{x}\left(\frac{\partial_{x} u^{2}-\partial_{x} v^{2}}{2}\right), u-v\right) \leq 4 N^{4} \frac{O_{1} O_{2}}{2}\|u-v\| .
$$

We will continue our investigation with the following case:

$$
\left(\varepsilon^{2} \partial_{x x x x}^{4}(u-v), u-v\right) .
$$

Without doubt, we can find four position real numbers, say, for instance, $\mathrm{O}_{3}, \mathrm{O}_{4}, \mathrm{O}_{5}$ and $\mathrm{O}_{4}$, allowing us to have the following relation:

$$
\left(\varepsilon^{2} \partial_{x x x x}^{4}(u-v), u-v\right) \leq \varepsilon^{2} O_{3} O_{4} O_{5} O_{4}\|u-v\|\|u-v\| .
$$

We can further use the fact that $(u, u),(v, v)<N^{2}$ to obtain

$$
\left(\varepsilon^{2} \partial_{x x x x}^{4}(u-v), u-v\right) \leq 2 N^{2} \varepsilon^{2} O_{3} O_{4} O_{5} O_{4}\|u-v\| .
$$

And also

$$
\left(\lambda \partial_{y y}^{2}(u-v), u-v\right) \leq 2 N^{2} \lambda O_{6} O_{7}\|u-v\| .
$$

However, by inserting (33), (32), and (29) into (23), we arrive at the following result:

$$
\begin{aligned}
& (B(u)-B(v), u-v) \\
& \quad \leq\left(2 N^{2} \varepsilon^{2} O_{3} O_{4} O_{5} O_{4}+2 N^{2} \lambda O_{6} O_{7}+4 N^{4} \frac{O_{1} O_{2}}{2}\right)\|u-v\| .
\end{aligned}
$$

It is obvious that we can take

$$
q=2 \mathrm{~N}^{2} \varepsilon^{2} \mathrm{O}_{3} \mathrm{O}_{4} \mathrm{O}_{5} \mathrm{O}_{4}+2 \mathrm{~N}^{2} \lambda \mathrm{O}_{6} \mathrm{O}_{7}+4 \mathrm{~N}^{4} \frac{\mathrm{O}_{1} \mathrm{O}_{2}}{2} .
$$

And hypothesis (H1) is verified.

It is also easier to prove hypothesis two just by realizing that

$$
\begin{gathered}
(B(u)-B(v), w) \leq P\|u-v\|\|w\|, \\
P=2 M^{2} \frac{O_{8} O_{9}}{2}+\varepsilon^{2} O_{10} O_{11} O_{12} O_{13}+\lambda O_{14} O_{15} .
\end{gathered}
$$

And then hypothesis (H2) is also verified. Therefore following with hypotheses (H1) and (H2) verified, we can without reservation establish the following theorem.

Theorem 5. Let us consider

$$
B(u)=\left(\partial_{x} u\right)^{2}+u \partial_{x x} u+\varepsilon^{2} \partial_{x x x x}^{4} u+\lambda \partial_{y y}^{2} u
$$

and consider the initial and boundary condition for (2); then the proposed method leads to a special solution of (2).

The proof of the above theorem follows directly from hypotheses (H1) and (H2) and also Lemma 3.

Theorem 6. Taking into account the initial conditions for (2), then the special solution of (2) $u_{\text {esp }}$ to which $u$ converges is unique. 


\section{Derivation of the Special Solution}

We make use of Algorithm 4 to derive the special solution for (2). Here we assume that the initial condition is given as

$$
u(x, y, 0)=x y .
$$

Then using the algorithm we obtain the following:

$$
\begin{aligned}
u_{1}(x, y, t)= & t^{\alpha}(-\operatorname{Sin}[2 y]+\operatorname{Sin}[2(x+y)] \\
& +2(x+\operatorname{Sin}[x] \operatorname{Sin}[x+2 y])) \\
\times & (4 \alpha \operatorname{Gamma}[\alpha])^{-1},
\end{aligned}
$$$$
u_{2}(x, y, t)
$$$$
=\frac{1}{24 \mathrm{Gamma}[1+\alpha]^{2}} t^{2 \alpha}
$$$$
\times(-3 \cos [x-y]+12 \cos [y]+4 \cos [3 y]
$$$$
-9 \cos [x+y]-7 \cos [3(x+y)]
$$$$
+3 \cos [x+3 y]+3 \sin [x-y]
$$$$
-36 \sin [y]-4 \sin [3 y]+39 \sin [x+y]
$$$$
+12 x(-2 \cos [2 y]+2 \sin [2 y]+\sin [x+y])
$$$$
+\sin [3(x+y)]
$$$$
+72 \sin [x](-\cos [x+2 y]+\sin [x+2 y])
$$$$
+3 \sin [x+3 y]) \text {, }
$$$$
u_{3(x, y, t)}
$$$$
=\frac{1}{96 \mathrm{Gamma}[1+\alpha]^{3}} t^{3 \alpha}
$$$$
\times 6\left(15+38 x+6 x^{2}-12 \cos [x]\right.
$$$$
-3 \cos [2 x]+4 \sin [x])
$$$$
+48 \cos [y]((-4+x)(-1+\cos [x])-(9+x) \sin [x])
$$$$
+4 \cos [2 y]\left(-189-135 x+48 x^{2}\right.
$$$$
+16 \cos [x]+(173-9 x) \cos [2 x]
$$$$
+8 \sin [x]+(-190+9 x) \sin [2 x])
$$$$
-24 \cos [3 y](-8+6 x+(3+2 x) \cos [x]+5 \cos [3 x]
$$$$
+(5+2 x) \sin [x]+35 \sin [3 x])
$$$$
+\cos [4 y](21+8 \cos [x]-29 \cos [4 x]+8 \sin [x]
$$$$
+18 \sin [2 x]-14 \sin [4 x])
$$$$
+48(8+3 x+(-8+x) \cos [x]+(11+x) \sin [x]) \sin [y]
$$$$
+4\left(186-153 x-48 x^{2}+4 \cos [x]\right.
$$

$$
\begin{aligned}
& +(-190+9 x) \cos [2 x]-20 \sin [x] \\
& +(-173+9 x) \sin [2 x]) \sin [2 y] \\
& -24(-40-6 x+(5+2 x) \cos [x]+35 \cos [3 x] \\
& -(3+2 x) \sin [x]-5 \sin [3 x]) \sin [3 y] \\
& +(8 \cos [x]+18 \cos [2 x]-2(6+7 \cos [4 x]+4 \sin [x]) \\
& +29 \sin [4 x]) \sin [4 y] \text {, } \\
& u_{4}(x, y, t) \\
& =\frac{1}{\text { 2880Gamma }[1+\alpha]^{4}} \\
& \times t^{4 \alpha}(-120 \cos [2 y] \\
& \times(1173+2 x(-351+x(-123+32 x)) \\
& -96 \cos [x]+3(-359+24 x) \cos [2 x] \\
& +24(2+x+6 x \cos [x]) \sin [x] \\
& -489 \sin [2 x]) \\
& +360(-39-36 x+(26+6 x) \cos [x] \\
& +(13+6 x) \cos [2 x] \\
& -2(1+x) \sin [x]-5 \sin [2 x]) \\
& -30 \cos [y](892-24(-5+x) x \\
& +12\left(-83+66 x+8 x^{2}\right) \cos [x] \\
& +84 \cos [2 x]+20 \cos [3 x] \\
& -3(-405+4 x(62+13 x)) \sin [x] \\
& +36 \sin [2 x]+15 \sin [3 x]) \\
& +5 \cos [3 y](8(-15883+18 x(-71+27 x)) \\
& +36(45+x(53+16 x)) \cos [x] \\
& +216 \cos [2 x] \\
& +4(31307-105 x) \cos [3 x] \\
& +9(-37+4 x(85+16 x)) \sin [x] \\
& +432 \sin [2 x] \\
& +5(-3665+12 x) \sin [3 x]) \\
& -180 \cos [4 y](111+56 x+12(7+x) \cos [x] \\
& +6 \cos [2 x]-201 \cos [4 x] \\
& +4(7+3 x) \sin [x] \\
& +2(13+6 x) \sin [2 x] \\
& +417 \sin [4 x])
\end{aligned}
$$




$$
\begin{aligned}
& +3 \cos [5 y](288+90 \cos [x]-150 \cos [3 x] \\
& -228 \cos [5 x]+105 \sin [x] \\
& +120 \sin [2 x]+200 \sin [3 x] \\
& -369 \sin [5 x]) \\
& -30((609+12 x(-41+3 x)) \cos [x] \\
& +36 \cos [2 x]+15 \cos [3 x] \\
& +12\left(-55+50 x+6 x^{2}\right. \\
& +(105+x(51+8 x)) \sin [x] \\
& -5 \sin [2 x]) \\
& -20 \sin [3 x]) \sin [y] \\
& +120\left(-453-630 x+330 x^{2}+64 x^{3}\right. \\
& +12(-3+x) \cos [x] \\
& +(489-72 x) \cos [2 x] \\
& +12(19+3 x) \sin [x] \\
& +3(-359+24 x) \sin [2 x]) \sin [2 y] \\
& +5(9(-51+4 x(85+16 x)) \cos [x] \\
& +432 \cos [2 x]+5(-3665+12 x) \cos [3 x] \\
& +4(4588-36 x(361+27 x) \\
& -9(48+x(53+16 x)) \sin [x] \\
& -54 \sin [2 x] \\
& +(-31307+105 x) \sin [3 x])) \sin [3 y] \\
& -180(4(7+3 x) \cos [x]+2(13+6 x) \cos [2 x] \\
& -3(157+12 x-139 \cos [4 x] \\
& +4(7+x) \sin [x]+2 \sin [2 x] \\
& -67 \sin [4 x])) \sin [4 y] \\
& +3(-56+105 \cos [x]+120 \cos [2 x] \\
& +200 \cos [3 x]-369 \cos [5 x] \\
& -90 \sin [x] \\
& +150 \sin [3 x]+228 \sin [5 x]) \sin [5 y]) \text {. }
\end{aligned}
$$

By using the proposed algorithm, one can obtain the remaining terms but for simplicity we stopped at 5 terms. The numerical solution is depicted in Figures 1 and 2 .

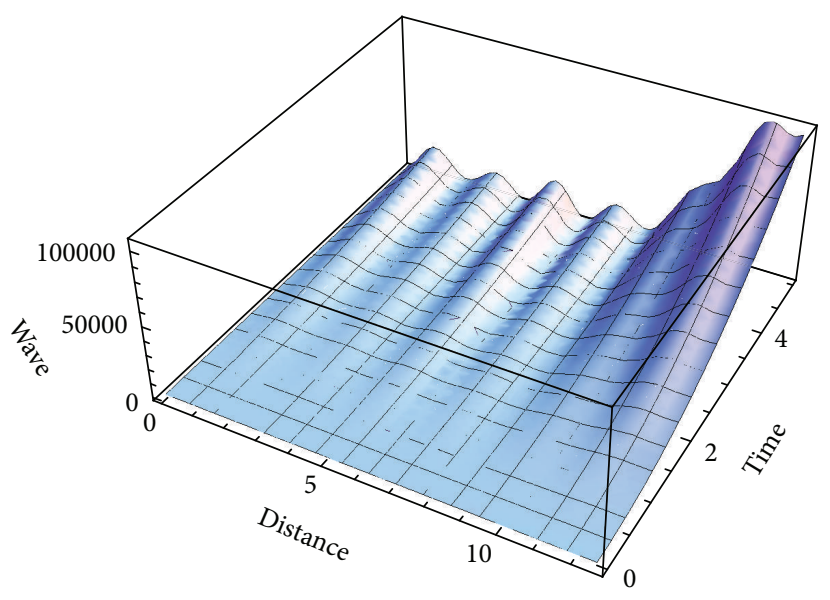

FIgURE 1: Solution for alpha equals 0.5 and $y=0$.

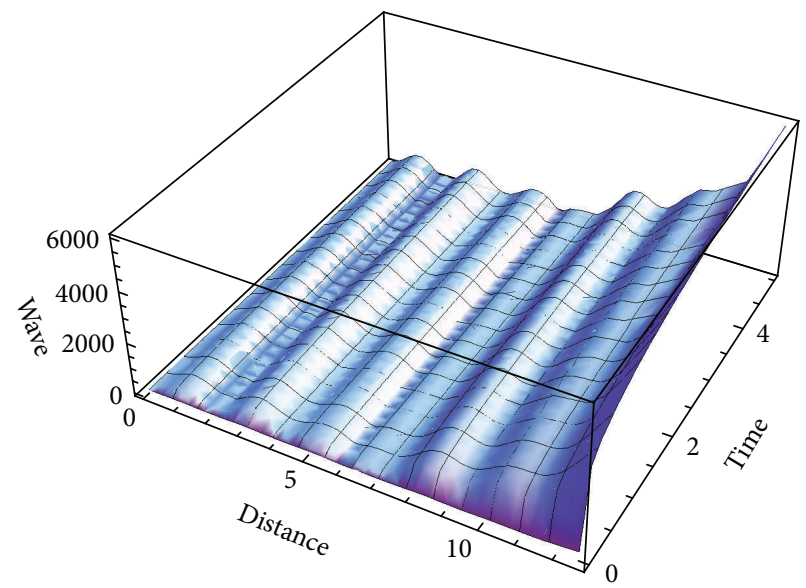

FIGURE 2: Solution for alpha equals 0.05 and $y=\mathrm{Pi}$.

\section{Conclusion}

In the recent years several physical phenomena were explained with great success in the light of the concept of noninteger order derivatives. More importantly to truthfully photocopy the nonlocal, frequency- and history-dependent possessions of power law phenomena, selected dissimilar modelling apparatuses constructed upon fractional operators have to be initiated. Especially, the remunerations of fractional calculus and fractional order models and their applications in the field of nonlinear wave motion have previously been intensively reconsidered during the last few eras with outstanding outcome. With this in mind, we have investigated and considered the motion of nonlinear wave within the folder of fractional derivative. A careful investigation of the stability, convergence, and uniqueness analysis has been done.

\section{Conflict of Interests}

The authors declare that there is no conflict of interests regarding the publication of this paper. 


\section{Authors' Contribution}

Abdon Atangana wrote the first draft and the other three authors corrected and submitted the final version.

\section{Acknowledgment}

Abdon Atangana would like to thank the Leon Claude Foundation for their financial support.

\section{References}

[1] M. J. Ablowitz and H. Segur, "On the evolution of packets of water waves," Journal of Fluid Mechanics, vol. 92, no. 4, pp. 691$715,1979$.

[2] M. J. Ablowitz and J. Villarroel, "On the Kadomtsev-Petviashvili equation and associated constraints," Studies in Applied Mathematics, vol. 85, no. 3, pp. 195-213, 1991.

[3] G. Biondini, "Line soliton interactions of the kadomtsevpetviashvili equation," Physical Review Letters, vol. 99, no. 6, Article ID 064103, 2007.

[4] G. Biondini and S. Chakravarty, "Soliton solutions of the Kadomtsev-Petviashvili II equation," Journal of Mathematical Physics, vol. 47, no. 3, Article ID 033514, 2006.

[5] G. Biondini and Y. Kodama, "On a family of solutions of the Kadomtsev-Petviashvili equation which also satisfy the Toda lattice hierarchy," Journal of Physics A: Mathematical and General, vol. 36, no. 42, pp. 10519-10536, 2003.

[6] A. Cloot and J. F. Botha, "A generalised groundwater flow equation using the concept of non-integer order derivatives," Water SA, vol. 32, no. 1, pp. 1-7, 2006.

[7] A. Atangana and P. D. Vermeulen, "Analytical solutions of a space-time fractional derivative of groundwater flow equation," Abstract and Applied Analysis, vol. 2014, Article ID 381753, 11 pages, 2014.

[8] A. Atangana and J. F. Botha, "Generalized groundwater flow equation using the concept of variable order derivative," Boundary Value Problems, vol. 2013, article 53, 2013.

[9] A. Atangana and N. Bildik, "The use of fractional order derivative to predict the groundwater flow," Mathematical Problems in Engineering, vol. 2013, Article ID 543026, 9 pages, 2013.

[10] A. Atangana and S. C. Oukouomi Noutchie, "Stability and convergence of a time-fractional variable order Hantush equation for a deformable aquifer," Abstract and Applied Analysis, vol. 2013, Article ID 691060, 8 pages, 2013.

[11] W. Deng, "Numerical algorithm for the time fractional FokkerPlanck equation," Journal of Computational Physics, vol. 227, no. 2, pp. 1510-1522, 2007.

[12] M. M. Meerschaert and C. Tadjeran, "Finite difference approximations for fractional advection-dispersion flow equations," Journal of Computational and Applied Mathematics, vol. 172, no. 1, pp. 65-77, 2004.

[13] C.-M. Chen, F. Liu, I. Turner, and V. Anh, "A Fourier method for the fractional diffusion equation describing sub-diffusion," Journal of Computational Physics, vol. 227, no. 2, pp. 886-897, 2007.

[14] C. Tadjeran, M. M. Meerschaert, and H.-P. Scheffler, "A secondorder accurate numerical approximation for the fractional diffusion equation," Journal of Computational Physics, vol. 213, no. 1, pp. 205-213, 2006.
[15] S. B. Yuste and L. Acedo, "An explicit finite difference method and a new von Neumann-type stability analysis for fractional diffusion equations," SIAM Journal on Numerical Analysis, vol. 42, no. 5, pp. 1862-1874, 2005.

[16] A. A. Kilbas, H. M. Srivastava, and J. J. Trujillo, Theory and Applications of Fractional Differential Equations, vol. 204 of North-Holland Mathematics Studies, Elsevier Science B.V., Amsterdam, The Netherlands, 2006.

[17] I. Podlubny, Fractional Differential Equations, vol. 198 of Mathematics in Science and Engineering, Academic Press, New York, NY, USA, 1999.

[18] A. Atangana and A. Secer, "A note on fractional order derivatives and table of fractional derivatives of some special functions," Abstract and Applied Analysis, vol. 2013, Article ID 279681, 8 pages, 2013.

[19] C. M. Bender and S. A. Orszag, Advanced Mathematical Methods for Scientists and Engineers. I, Springer, New York, NY, USA, 1999.

[20] P. K. Jain and K. Ahmad, Definitions and Basic Properties of Inner Product Spaces and Hilbert Spaces, Functional Analysis, New Age International, 2nd edition, 1995.

[21] E. Prugovečki, Quantum Mechanics in Hilbert Space, vol. 92 of Pure and Applied Mathematics, Academic Press, New York, NY, USA, 2nd edition, 1981.

[22] G. B. Arfken and H. J. Weber, Mathematical Methods for Physicists, Academic Press, Boston, Mass, USA, 5th edition, 2001. 


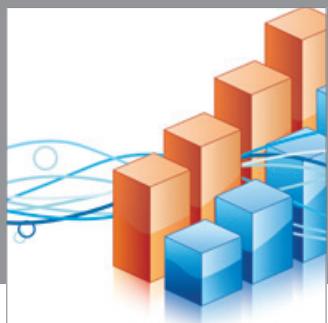

Advances in

Operations Research

mansans

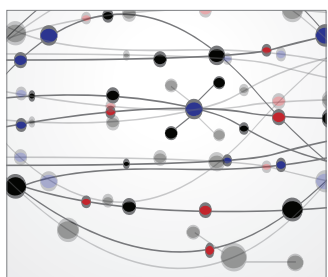

The Scientific World Journal
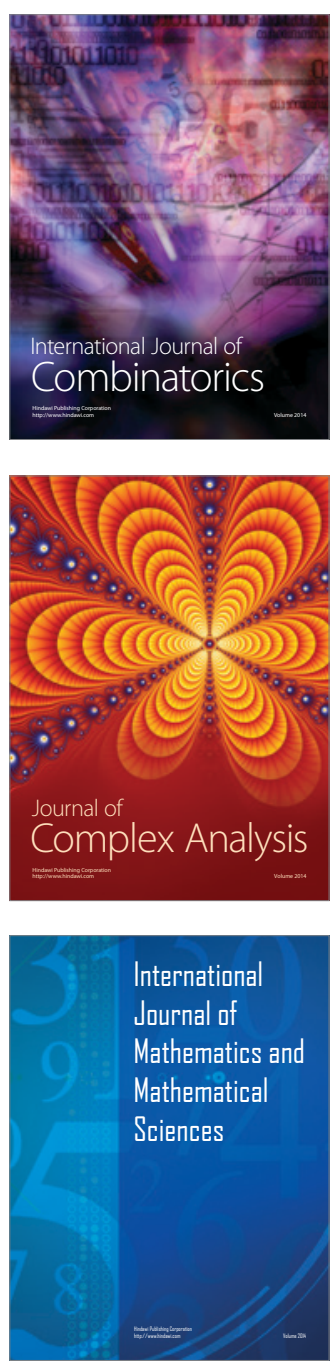
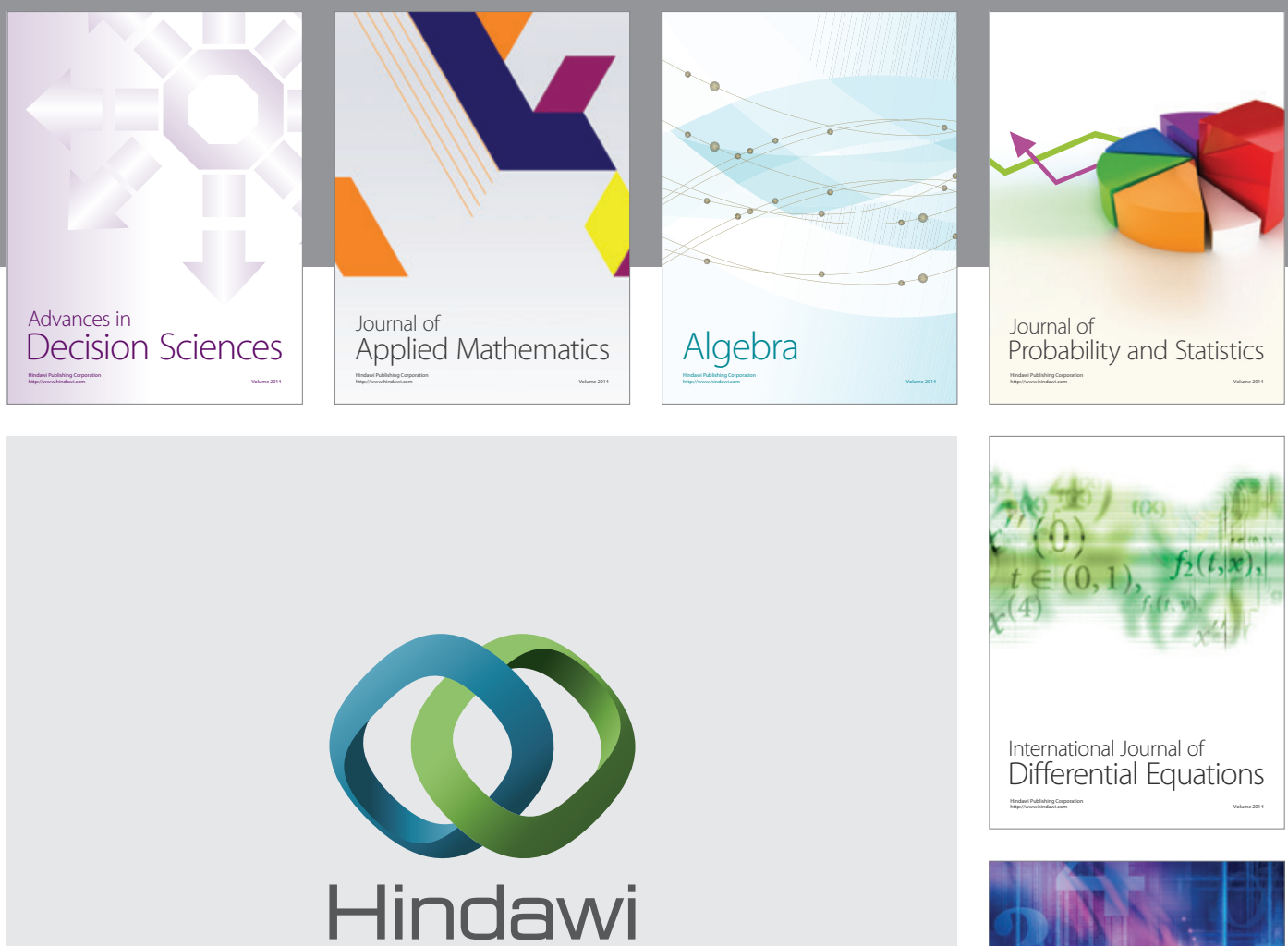

Submit your manuscripts at http://www.hindawi.com
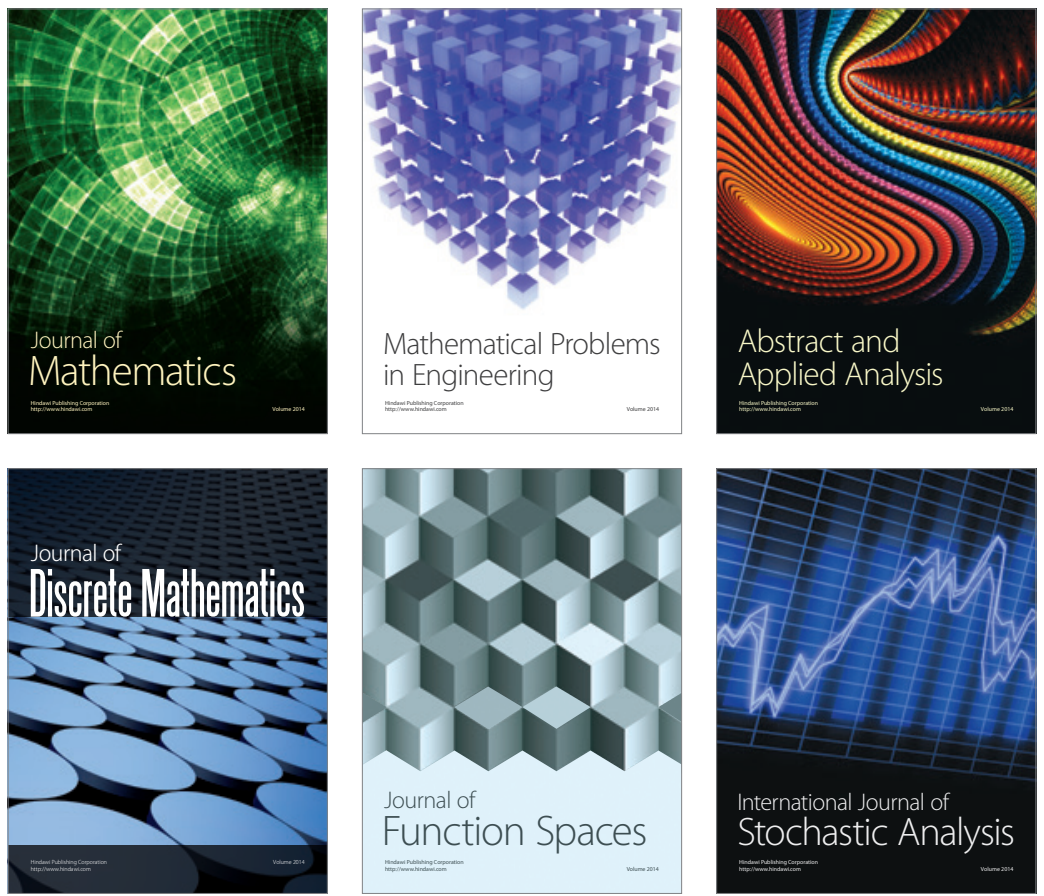

Journal of

Function Spaces

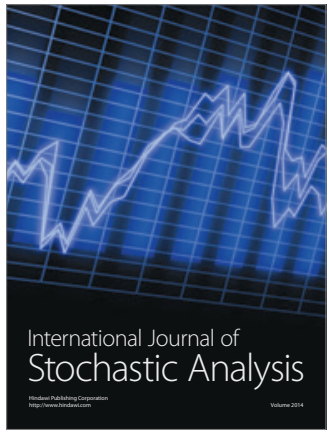

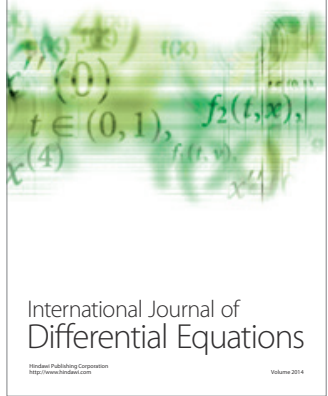
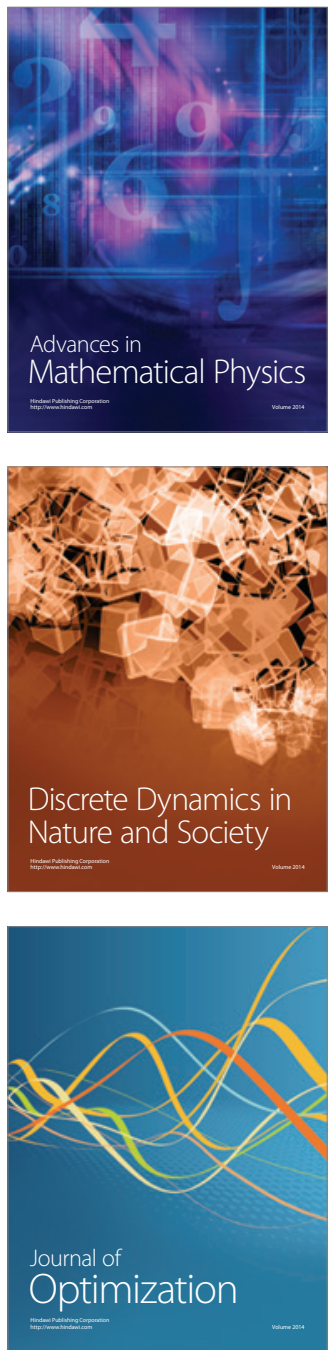